\section{LANTHANUM DEPOSITION IN THE GASTRIC MUCOSA}

Victoria Van Winden, Sheng Khor

Anatomical Pathology Department, PathWest, Perth, WA,

Australia

Lanthanum carbonate is a phosphate binding agent used for the management of hyperphosphataemia in patients with chronic renal failure. Cases of lanthanum deposition within the gastrointestinal tract, first recognised in gastric mucosa, have been reported since 2015. However, lanthanum deposition remains a relatively novel entity with potential for under-recognition and confusion with a variety of other processes. This case study reports a case of lanthanum deposition involving the gastric mucosa in a renal transplant patient. Biopsies taken from the gastric antrum demonstrated appearances of reactive gastropathy together with an accumulation of histiocytes, including occasional multinucleated forms, containing deposits of amphophilic to brown finely granular to coarse material within the superficial lamina propria. The differential diagnosis included gastric mucosal calcinosis, iron therapy-induced gastric injury, an infectious aetiology, or the accumulation of exogenous materials related to medications including resins/ crystalloids. Investigation of the patient's medication history revealed oral lanthanum carbonate use; this finding taken in conjunction with the morphologic findings was most in keeping with lanthanum deposition. The identification of lanthanum deposition in gastric biopsies is important as the long-term consequences are currently unknown, and the use of this medication is increasing.

\section{HAEMOSIDEROTIC FIBROLIPOMATOUS TUMOUR - A CASE REPORT}

Mthulisi Viki, Gelareh Farshid

Anatomical Pathology, SA Pathology, Adelaide, SA, Australia

In our case we describe a 46-year-old woman who presented with 6 months of right dorsal foot swelling. Examination revealed sharp aching pain over the dorsal first metatarsophalangeal joint. Multi-modality imaging with plain radiography, CT and ultrasound showed a heterogenous solid subcutaneous mass of the dorsal forefoot. Subsequent MRI reported dilated slow flow vascular spaces interposed with fat, favouring haemangioma. Core biopsy performed favoured spindle cell lipoma.

Progressive growth of the lesion with functional impairment led to excision at 18 months follow up. Sections of the excision specimen showed mature fat with spindle cell proliferation and myxoid areas. Fibrotic and collagenous appearance were identified. The spindled cells formed fascicles and nodular aggregates between the adipocytes. Spindle cells were atypical and showed haemosiderin staining in keeping with haemosiderotic fibrolipomatous tumour (HFLT)

HFLT is a rare mesenchymal tumour. As seen in this case, it is locally aggressive with an associated $30-50 \%$ recurrence rate. Immunohistochemistry of the spindle cell component is typically positive for CD34 and calponin. The chromosomal translocation $\mathrm{t}(1 ; 10)(\mathrm{p} 22 ; \mathrm{q} 24)$ with the fusion gene TGFBR3-MGEA5 is associated with this lesion, as well as pleomorphic hyalinising angiectatic tumours and myxoinflammatory fibroblastic sarcoma.

\section{HUNTING FOR SNAKES AND RUBIES}

Sophie Walter ${ }^{1,2,3}$, Simone L. Van Es ${ }^{2}$, Peter Earls ${ }^{3}$, ${ }_{\text {Raquel Alvarado }}^{1}$, Janet Rimmer ${ }^{1,4,5}$, Richard Harvey ${ }^{1,6}$ ${ }^{1}$ Rhinology and Skull Base Research Group, St Vincent's Centre for Applied Medical Research, University of New South Wales, Sydney, Australia; ${ }^{2}$ Department of Pathology, School of Medical Sciences, Faculty of Medicine, University of New South Wales, Sydney, Australia; ${ }^{3}$ SydPath, St Vincent's Hospital, Sydney, Australia; ${ }^{4}$ Woolcock Institute, University of Sydney, Sydney, Australia; ${ }^{5}$ Faculty of Medicine, Notre Dame University, Sydney, Australia; and ${ }^{6}$ Faculty of Medicine and Health Sciences, Macquarie University, Sydney, Australia

Background: Digital pathology is increasingly part of diagnostic workflow. ${ }^{1}$ RCPA Anatomical Pathology small biopsy slide exam already uses whole slide images (WSI), with plans to similarly digitise the cytopathology slide exams. Granulocytes are historically difficult to assess on WSI. ${ }^{1}$ Chronic rhinosinusitis (CRS) is a heterogenous inflammatory condition. CRS subtype is diagnosed via histopathology. ${ }^{2}$ Targeted biologic therapies are being studied for eosinophilic CRS, the most common and severe subtype in Australia, and accurate endotyping is important. Thus, on glass slides and WSI, differentiating eosinophils, with their bilobed nuclei and ruby-red cytoplasmic granules, from neutrophils, with their snake-like multilobate nuclei, is required to guide management.

Aims: To quantify eosinophil number on sinonasal tissue WSI, and validate WSI against the equivalent glass slides.

Methodology: On WSI, eosinophils were independently counted by two independent assessors. An anatomical pathologist validated eosinophil counts on WSI against equivalent glass slides after a washout period.

Results: Interobserver scoring for the assessors of tissue eosinophil count on WSI demonstrated excellent agreement [intraclass correlation coefficient (ICC) $0.963, p<0.001]$. Intraobserver tissue eosinophil count on WSI and the glass slide indicated excellent agreement (ICC 0.988, $p=0.009$ ).

Conclusion: Eosinophil morphology and colour were accurately and consistently identified on WSI

Acknowledgements:

The Royal College of Pathologists of Australasia Quality Assurance Programs (RCPAQAP) hosted the WSIs. Ms Stephanie Gay, from RCPAQAP, digitalised the histopathological slides. Ms Tanya Wyatt helped with tissue embedding.

\section{References}

1. Van Es SL. Digital pathology: semper ad meliora. Pathology 2019; 51: $1-10$.

2. Snidvongs K, Lam M, Sacks R, et al. Structured histopathology profiling of chronic rhinosinusitis in routine practice. Int Forum Allergy Rhinol 2012; 2: 376-85.

\section{A DECEPTIVE CASE OF GASTRIC CARCINOMA WITH LYMPHOID STROMA}

A. Walton, A. Sinha

${ }^{I}$ Department of Anatomical Pathology, Pathology Queensland, The Townsville Hospital, Townsville, Australia

Gastric carcinoma with lymphoid stroma is a rare variant of gastric carcinoma characterised by tumour cells dispersed within a lymphocytic background. ${ }^{1}$ This case reports a 67 -year-old male 